\title{
Adaptación y Validación al Español de la Escala de Compromiso Profesional y Aspiraciones de Desarrollo Profesional
}

\author{
Adaptation and Validation into Spanish of the Professional Engagement and Career \\ Development Aspirations
}

\author{
Pedro Antonio Sánchez-Miguel ${ }^{1}$, Diana $\mathrm{Amado}^{2}$, Santiago Mendo-Lázaro $^{3}$, Benito León-Del Barco ${ }^{3}$ \\ y Francisco Miguel Leo ${ }^{1}$
}

\begin{abstract}
Resumen
El desarrollo profesional es el apoyo formal e informal de las actividades que son designadas para ayudas a los profesores a su desarrollo como docente. El objetivo de esta investigación fue validar la escala de compromiso profesional y aspiraciones de desarrollo profesional en una muestra de maestros de infantil, primaria y profesores de enseñanza secundaria en España. La muestra estuvo formada por 376 maestros y profesores, pertenecientes a enseñanza infantil $(n=136)$, primaria $(n=159)$ y secundaria $(n=71)$, tanto de género masculino $(n=110)$ como femenino $(n=266)$, y de centros públicos $(n=269)$, concertados $(n=106)$ y privados $(n=1)$ de la Región Extremadura. Los resultados obtenidos mostraron que el instrumento presenta una adecuada validez factorial y consistencia interna. Se concluye que este trabajo aporta evidencias científicas de que la escala de compromiso profesional y aspiraciones de desarrollo profesional se muestra válida y fiable para analizar esos comportamientos en una muestra de maestros y profesores y maestros españoles.
\end{abstract}

Palabras clave: maestros, evaluación, propiedades psicométricas, desarrollo laboral

\begin{abstract}
Professional development is the formal and informal support and activities that are designed to help teachers develop as professionals. The aim of this research was to validate the professional engagement and career development aspirations in a sample size of teachers from Primary and Secondary schools from Spain. The sample was formed by 376 teachers belonged to Childhood $(n=136)$, Primary $(n=159)$ and Secondary $(n=71)$, both male $(n=110)$ and female $(n=266)$, from public $(n=269)$, state $(n=106)$ and private $(n=1)$ schools from the Region of Extremadura. Results showed that the instrument had an adequate factorial validity and internal consistency. Finally, this work gives empiric evidences that the professional engagement and career development aspirations is valid and reliable to examine these behaviors in teachers.
\end{abstract}

Keywords: teachers, evaluation, psychometrics properties, career development

\footnotetext{
${ }^{1}$ Departamento de Didáctica de la Expresión Musical, Plástica y Corporal. Facultad de Formación del Profesorado. Universidad de Extremadura. Avenida de la Universidad, S/N, 10071. Cáceres, España. Correo: pesanchezm@unex.es; franmilema@unex.es

${ }^{2}$ Departamento de Educación Física. Facultad de Ciencias Sociales y Jurídicas. Universidad Rey Juan Carlos. Paseo de los Artilleros, 0, 28032, Madrid, España. Correo: diamal@unex.es

${ }^{3}$ Departamento de Psicología y Antropología. Facultad de Formación del Profesorado. Universidad de Extremadura. Avenida de la Universidad, S/N, 10071. Cáceres, España. Correo: smendo@unex.es; bleon@unex.es

Correspondencia: Pedro Antonio Sánchez-Miguel. Departamento de Didáctica de la Expresión Musical, Plástica y Corporal. Facultad de Formación del Profesorado. Universidad de Extremadura. Avenida de la Universidad, S/N, 10071. Cáceres, España. Tel.: 927257049. Correo: pesanchezm@unex.es
} 


\section{Introducción}

El entorno educativo necesita de profesionales que constantemente adapten sus conocimientos y destrezas a los requerimientos actuales de la profesión. En este sentido, los profesores son profesionales que requieren de una alta capacitación y responsabilidad para conseguir en el alumnado el mejor rendimiento académico, así como alcanzar las expectativas marcadas desde el inicio (OECD, 2009). Las líneas de investigación han tratado de comprobar las motivaciones de los profesores, sus creencias y su desarrollo profesional temprano, con el objetivo de conocer las razones que llevan a los individuos a ser profesores, cómo perciben su profesión, así como las aspiraciones profesionales que tienen (Malderez, Hobson, Tracey, \& Kerr, 2007; Richardson \& Watt, 2005; Rinke, 2008; Wang, 2004; Watt \& Richardson, 2008).

Entre los factores que conducen a la elección de ser profesor, está bien documentado que una de las principales razones es la motivación, sugiriendo el carácter multidimensional y no unidimensional de este constructo (Spittle, Jackson, \& Casey, 2009). En esta línea, los estudios científicos nos señalan una motivos económicos, sociales, de posible movilidad geográfica, interpersonales, intelectuales, tiempo para las familias, status social, beneficios derivados del trabajo como seguridad, pensiones, vacaciones, así como la percepción de habilidades relativas al desempeño correcto de la profesión de profesor/maestro (Boz \& Boz, 2008; Malderez et al., 2007; Wang, 2004; Watt \& Richardson, 2007), entre las razones fundamentales por las que se optan por elegir la carrera docente.

De esta manera, debido a la enorme heterogeneidad que muestran los maestros y profesores para acceder a su profesión, parece adecuado indicar que puede haber diferentes "tipos" de profesores, quienes, en función del perfil que posean, en relación con el tiempo dedicado a conseguir su puesto de trabajo, el grado en el que estos profesores están comprometidos con sus posibilidades de aprendizaje, sus aspiraciones de liderazgo en los colegios, así como el grado de satisfacción en el desempeño de su labor profesional ... optan por la labor docente como desarrollo profesional (Watt
\& Richardson, 2008). Por el contrario, existen motivos por los que los profesores desean no continuar con su labor docente. Entre estos factores destacan el bajo salario que tienen a pesar del trabajo que desempeñan, la falta de aspiraciones, y una pequeña proporción de profesores señalan experiencias negativas durante su labor en los centros educativos (Watt \& Richardson, 2008). Entre todos estos factores, se destaca la falta de satisfacción en la labor docente, debido fundamentalmente a altas cargas de trabajo y/u otros tipos de presión (DeAngelis \& Presley, 2011).

Esto último puede suponer una nueva perspectiva de trabajo, ya que podría ayudar a explicar los posibles abandonos en las primeras etapas de la carrera docente, siendo este aspecto todavía no estudiado en educación (Watt, Richardson, \& Wilkins, 2014). Por otro lado, Coldwell (2016) halló que el desarrollo profesional en las mujeres está limitado por su deseo de crear una familia, lo que supone un obstáculo para su desarrollo, teniendo que optar muchas veces por reducir su jornada laboral, o directamente optar a excedencias que impidan su capacidad de liderazgo y/o desarrollo. Otros aspectos como el carácter interino o funcionarial, se ha mostrado importante para continuar en una formación constante dentro de una actividad profesional (Richter, Kunter, Klusmann, Lüdtke, \& Baumert, 2011).

En este sentido, Anthony y Ord (2008) estudiaron qué o quién motivaba al individuo a cambiar su labor actual por la carrera docente, y hallaron que las percepciones de los profesores sobre su desarrollo profesional se podrían encuadrar en tres perfiles diferentes: "perfil bueno" (consistente en aquellos que seleccionaron la docencia por razones pragmáticas como seguridad en el trabajo y tiempo para la familia), "el tiempo es un derecho" (formado por aquellos que tenían un fuerte deseo por la docencia), "la enseñanza lo es todo para mí" (compuesto por aquellos que estaban atraídos hacia la docencia gracias a las experiencias positivas y aprendizajes que adquirían). Los resultados del trabajo mostraron que aquellos profesores que se encuadraban dentro del perfil "la enseñanza lo es todo para mí", eran los que estaban más satisfechos con la docencia, y tenían mayores 
índices de persistencia en el trabajo. Por otro lado, Mau y Mau (2006) estudiaron la persistencia en el trabajo en una investigación longitudinal de cuatro años y en una muestra amplia de docentes $(\mathrm{n}=24000)$, hallando que un $40 \%$ de los estudiantes persistían en sus aspiraciones docentes después de haber iniciado el proceso a ser profesor/maestro, mientras que el porcentaje restante $(60 \%)$ pertenecía a aquellos estudiantes que habían abandonado su carrera para llegar a ser docentes. Además, los autores destacaron que aquellos estudiantes que persistían eran los que obtuvieron inicialmente mejores rendimientos económicos, tenían un mayor locus de control, así como provenían de un estatus socioeconómico más alto, y también tenían padres con un mayor nivel académico e implicación en su formación.

En la misma línea, autores como Bruinsma y Jansen (2010) testaron la relación entre las motivaciones para ser docentes y las intenciones para continuar siéndolo en una muestra de profesores holandeses. Los autores encontraron que los profesores con motivos extrínsecos menos adaptativos (i.e. enseñanza como último recurso) intentaban permanecer en la enseñanza menos años que aquellos que tenían motivos intrínsecos más adaptativos (i.e. trabajar con niños) y motivos extrínsecos adaptativos (i.e. buenas oportunidades profesionales). Al respecto, otros autores como Day y $\mathrm{Gu}$ (2007) indicaron que aquellos profesores que tenían un alto desarrollo profesional en sus carreras, enfatizando el carácter emocional que supone la enseñanza, eran aquellos que persistían más en la misma, además de tener un mayor compromiso hacia el aprendizaje en su tarea profesional, y presentar comportamientos más positivos hacia alumnos y enseñanza en general. Finalmente, Coldwell (2017) agrupó todos los factores y/o consecuencias posibles que determinan la elección de la profesión docente, y sus consecuencias para continuar y/o abandonar, generando un modelo predictivo que ayuda a entender los diferentes procesos en el profesorado, generando un marco conceptual válido para el tratamiento del desarrollo profesional.

En este sentido, la importancia de validar un instrumento aplicable en el contexto de la educación radica en la relevancia de las consecuencias en el proceso de enseñanza y aprendizaje hacia el alumnado. Así, Atxurra,
Villardón-Gallego y Calvete (2015) diseñaron una escala para valorar el grado de aplicación del aprendizaje cooperativo en las aulas universitarias. Otros autores como ÁlvarezGarcía, Núñez, Rodríguez, Álvarez y Dobarro (2011) han diseñado una escala para analizar la aparición de comportamientos violentos en el contexto educativo, tanto por parte de los alumnos, como por parte del profesor. Por otro lado, González-Lomelí, Maytorena, CárdenasNiño y Tapia-Fonllem (2018) analizaron las orientaciones temporales en alumnos colombianos, generando un instrumento válido $\mathrm{y}$ fiable en el contexto educativo. Castillo, Solano y Sepúlveda (2018) validaron al contexto mexicano la escala de actitudes masculinas sobre el cuerpo en estudiantes varones universitarios, mostrando unas propiedades psicométricas adecuadas. Sin embargo, y hasta dónde se conoce, se destaca no se han diseñado ni validado ninguna escala que mida el compromiso profesional y aspiraciones de desarrollo profesional del profesorado de forma científica, mediante análisis factoriales confirmatorios del modelo teórico del constructo en el contexto español.

Basado en los estudios mostrados, esa investigación se centra en el individuo como medio de acercamiento tipológico de análisis, y que consistente con las teorías modernas de desarrollo, dónde el individuo se considera como una unidad de organización en su desarrollo (Roeser \& Galloway, 2002). De esta manera, los análisis centrados en el individuo poseen un buen ajuste metodológico con una buena perspectiva holística sobre el desarrollo humano (Roeser \& Galloway, 2002).

Por todo ello, y debido a la importancia que tiene en la actualidad el conocer aspectos de compromiso y desarrollo profesional, el objetivo de este trabajo es validar la escala de compromiso profesional y aspiraciones de desarrollo profesional en una muestra de maestros de infantil y primaria y profesores de enseñanza secundaria en España, generando un instrumento riguroso y científico en el contexto educativo (Desimone, 2009). En base al objetivo, la hipótesis que planteamos es que la validación de la escala de compromiso profesional y aspiraciones de desarrollo profesional presentará una adecuada 
validez y fiabilidad en una muestra de maestros y profesores españoles.

\section{Método}

\section{Participantes}

La muestra estuvo formada por 376 maestros y profesores, con edades comprendidas entre los 26 y los 64 años $(M=41.54 ; D T=9.02)$, y entre 1 y 39 años de experiencia docente $(M=16.54$; $D T=9.16)$, pertenecientes a enseñanza infantil $(n=136)$, primaria $(n=159)$ y secundaria $(n=71)$, tanto de género masculino $(n=110)$ como femenino ( $n=266)$, y de centros públicos $(n=269)$, concertados $(n=106)$ y privados $(n=1)$ de la Comunidad Autónoma de Extremadura. La selección de la muestra se realizó por conglomerados estratificado.

\section{Procedimiento}

Una que vez que el proyecto de desarrollo del instrumento en el idioma original tenía como objetivo la traducción y adaptación al español, en la etapa de construcción del instrumento se aplicó el método decentering ${ }^{l}$ (Brislin, 1970). Este método tuvo como objetivo facilitar la traducción del instrumento a otros idiomas $y$, consecuentemente, la generalización de los instrumentos de medida. En la fase de traducción de la versión original, participó un investigador internacional bilingüe, colaborando en todas las fases de desarrollo del cuestionario de escala de compromiso profesional y aspiraciones de desarrollo profesional. Se siguieron las pautas de la Comisión Internacional de Test (ITC) para traducción y adaptación de instrumentos en el ámbito educacional y psicológico (Hambleton, 2005), tanto para el decentering (dónde se verificaron las palabras o expresiones sin traducción equivalente, con diferente significado

\footnotetext{
${ }^{1}$ Decentering se refiere al método utilizado para palabras o expresiones que no tienen equivalencia en el idioma (idioma al cual se pretende traducir y adaptar el instrumento. La versión original (source version) así como la versión traducida son igualmente importantes, por eso las revisiones y cambios pueden ser realizadas en la versión original, en el caso que se pueda hacer los dos procesos - desarrollo de la escala y la traducción - en el mismo periodo temporal (Brislin, 1970).
}

connotativo o que simplemente no existían en español (Muñiz, Elosua, \& Hambleton, 2013), como la traducción equivalente (dónde se proporcionó garantías de equivalencia conceptual, lingüística y métrica (Daouk \& McDowall, 2013; Hambleton, 2001), la retro-traducción (dónde un experto tradujo de nuevo los items en español obtenidos en la fase anterior al inglés), y la versión consensuada (los autores del cuestionario de escala de compromiso profesional y aspiraciones de desarrollo profesional y el investigador bilingüe analizaron la retrotraducción en conjunto).

La primera versión fue revisada por dos psicólogos bilingües expertos en validación de cuestionarios. Una vez realizada la traducción, fue revisada a través de un juicio de expertos en la temática, quienes recibieron la versión original en inglés y la versión traducida en español. Estos expertos examinaron y testaron la comprensión de los ítems redactados en español y su equivalencia en contenido con los originales. Así, en todos aquellos ítems que no se alcanzó un grado de acuerdo de al menos el $85 \%$ sobre la comprensión o equivalencia del contenido, se llevaron a cabo las sugerencias dadas por los expertos. Finalmente, la escala en español fue entregada a 10 profesores universitarios, 10 profesores de enseñanza secundaria y 10 profesores de enseñanza infantil y primaria, para testar la comprensión de los ítems. Más adelante, y tras comprobar la comprensión de los ítems, se obtuvo la versión definitiva que se ha empleado en la presente investigación.

El estudio recibió el consentimiento del Comité Ético de la Universidad de Extremadura. Todos los participantes fueron tratados en condiciones de igualdad siguiendo la guía ética de la Asociación Americana de Psicología con respecto al consentimiento, confidencialidad y anonimato en las respuestas.

Previamente a realizar el estudio, se informó a los participantes de la voluntariedad de la investigación, al igual que del tratamiento confidencial de los datos. Con respecto a la recogida de datos, se realizó un protocolo de actuación con el objetivo de que fuera similar durante todo el proceso. En primer lugar, el investigador principal se puso en contacto con los maestros y profesores de los diferentes centros 
educativos objeto de estudio. Estos profesores eran los tutores de prácticas de los alumnos de infantil, primaria y secundaria que realizaban las prácticas en los diferentes centros de enseñanza (asignaturas correspondientes a los Grados de Educación Infantil, Primaria y Máster de Enseñanza Secundaria Obligatoria). Para ello, se facilitó un escrito informativo sobre los objetivos de la investigación, así como un consentimiento informado de ello. Una vez aceptado por parte de los profesores, se administró al alumnado la escala que debían rellenar.

La administración de la escala se llevó a cabo en horario escolar, ofreciendo las instrucciones pertinentes a los maestros y profesores mediante una pequeña guía que el investigador principal facilitó al alumnado. Se insistió en el carácter anónimo de la investigación. La escala fue completada de manera individual y en un clima adecuado, que les permitía concentrarse sin tener ningún tipo de distracción. El proceso de duró aproximadamente 15 minutos.

\section{Instrumentos}

Escala de compromiso profesional y aspiraciones de desarrollo profesional (PECDA) (Watt \& Richardson, 2008). La Escala PECDA constituida por 17 ítems, incluye cuatro factores: esfuerzo previsto (4 ítems, i.e.: “¿Con qué intensidad te vas a esforzar para ser un maestro/profesor eficaz?”), persistencia planificada (4 ítems, i.e.: “¿Qué seguridad tienes de persistir en la enseñanza?"), aspiración de desarrollo profesional (5 ítems, i.e.: “¿Cuánto deseas participar en cursos para tu desarrollo profesional?"), y las aspiraciones de liderazgo (4 ítems, i.e.: “¿Cuánto deseas buscar un rol de supervisión/gestión en tu centro educativo?"). (Ver Anexo). Las respuestas se realizaban en una escala tipo Likert de 5 puntos, dónde el 1 se correspondía con nada y el 5 se correspondía con extremadamente.

\section{Análisis de los datos}

Se calcularon los estadísticos descriptivos y los valores de asimetría y curtosis de cada uno de los ítems que componen el cuestionario para comprobar la normalidad univariada de los ítems. A continuación, se llevó a cabo un análisis de las propiedades psicométricas de la escala de compromiso profesional y aspiraciones de desarrollo profesional, utilizando el análisis factorial confirmatorio mediante el programa AMOS 21. Para testar el ajuste entre el modelo teórico planteado y la matriz de datos recogida se utilizaron diferentes índices: $\chi^{2} / g l$ (Chi-Square / degrees of freedom), CFI (Comparative Fit Index), TLI (Tucker Lewis Index), RMSEA (Root Mean Square Error of Approximation) y SRMR (Standardized Root Mean Residual). Mediante el cálculo de la varianza media extractada, la fiabilidad compuesta y el omega de McDonald, se evaluó la consistencia interna de la escala de adaptación del PECDA (escala de compromiso profesional y aspiraciones de desarrollo profesional). Además, se realizó un análisis de la validez nomológica a través de un análisis de correlaciones bivariadas con el coeficiente de Pearson y un análisis multivariado (MANOVA) utilizando para ello el programa SPSS 21.

\section{Resultados}

Se dividió la muestra original ( $n$ 376) en dos submuestras extraídas aleatoriamente $\left(n_{l}=188\right.$ y $\left.n_{2}=188\right)$. La primera $\left(n_{1}\right)$ se utilizó para realizar el análisis factorial exploratorio (AFE) y la segunda $\left(n_{2}\right)$ como muestra de validación en el análisis factorial confirmatorio (AFC). Ambas submuestras eran equivalentes en función del género $[\chi 2(1)=.151, p=.698]$ y del nivel de enseñanza educativa (docentes de Educación Infantil, Primaria y Secundaria) $[\chi 2(2)=4.394$, $p=.222]$.

\section{Análisis factorial exploratorio}

La medida de adecuación muestral $(\mathrm{KMO}=.847)$ y la prueba de esfericidad de Bartlett $\left(\chi^{2}=3173,095(136), p<.001\right)$, indicaron que tenía sentido realizar el análisis factorial. Mediante el modelo de componentes principales con rotación Oblimin, se obtiene una solución de cuatro factores (Tabla 1), que explican el $68.75 \%$ de la varianza. El factor 1, esfuerzo previsto (4 ítems), explica el $33.81 \%$ de la varianza. El factor 2, aspiraciones de liderazgo (4 ítems), explica el $16.93 \%$ de la varianza. El factor 3, persistencia planificada (4 ítems), explica el $11.84 \%$ de la 
Tabla 1. Análisis factorial exploratorio de la escala PECDA. Análisis de componentes principales, rotación Oblimin

\begin{tabular}{|c|c|c|c|c|c|c|c|}
\hline \multirow[b]{2}{*}{$\begin{array}{l}\text { Factor } \\
(\text { PECDA) }\end{array}$} & \multirow[b]{2}{*}{$M$} & \multirow[b]{2}{*}{$D T$} & \multicolumn{4}{|c|}{ Ítems de la escala de compromiso profesional y aspiraciones de desarrollo $\ldots$ Componente } & \multirow[b]{2}{*}{$\begin{array}{l}\text { Comuna- } \\
\text { lidades }\end{array}$} \\
\hline & & & $\begin{array}{l}\text { Items de la escala de compromiso protesional y aspiraciones de desarrollo - } \\
\text { profesional }\end{array}$ & 1 & 2 & 4 & \\
\hline \multirow{4}{*}{$\begin{array}{l}\text { Esfuerzo } \\
\text { previsto } \\
\text { (Varianza } \\
\text { explicada } \\
33.81 \% \text { ) }\end{array}$} & 4.71 & .54 & $\begin{array}{l}1 \text { ¿Con qué intensidad te vas a esforzar para ser un maestro/profesor } \\
\text { eficaz? }\end{array}$ & .928 & & & .863 \\
\hline & 4.73 & .52 & 2 ¿Cuánto tienes pensado esforzarte como maestro/profesor? & .910 & & & .833 \\
\hline & 4.67 & .56 & 3 ¿Cuánto trabajarás para ser un buen maestro/profesor? & .858 & & & .743 \\
\hline & 4.68 & .55 & 4 ¿Cuánto esfuerzo pondrás en tu enseñanza? & .853 & & & .732 \\
\hline \multirow{4}{*}{$\begin{array}{l}\text { Aspiraciones } \\
\text { de liderazgo } \\
\text { (Varianza } \\
\text { explicada } \\
16.93 \% \text { ) }\end{array}$} & 2.30 & 1.14 & $\begin{array}{l}5 \text { ¿Cuánto deseas buscar un rol de supervisión/gestión en tu centro } \\
\text { educativo? }\end{array}$ & & .882 & & .784 \\
\hline & 2.43 & 1.17 & $\begin{array}{l}6 \text { ¿Cuánto deseas tener responsabilidades de liderazgo en tu centro } \\
\text { educativo? }\end{array}$ & & .867 & & .760 \\
\hline & 2.30 & 1.55 & $\begin{array}{l}7 \text { ¿Cuánto deseas alcanzar una posición de gestión en tu centro } \\
\text { educativo? }\end{array}$ & & .816 & & .678 \\
\hline & 2.30 & 2.45 & 8 ¿Cuánto deseas iniciar un rol de liderazgo en tu centro educativo? & & .617 & & .386 \\
\hline \multirow{4}{*}{$\begin{array}{l}\text { Persistencia } \\
\text { planificada } \\
\text { (Varianza } \\
\text { explicada. } \\
11.84 \% \text { ) }\end{array}$} & 4.42 & .80 & 9 ¿Qué certeza tienes que vas a permanecer en la enseñanza? & & & .927 & .867 \\
\hline & 4.37 & .83 & $\begin{array}{l}10 \text { ¿Qué seguridad tienes que vas a permanecer en el puesto de } \\
\text { maestro/profesor? }\end{array}$ & & & .890 & .798 \\
\hline & 4.50 & .74 & 11 ¿Qué seguridad tienes de persistir en la enseñanza? & & & .848 & .735 \\
\hline & 4.53 & .75 & 12 ¿Qué confianza tienes que vas a seguir unido la enseñanza? & & & .755 & .613 \\
\hline \multirow{5}{*}{$\begin{array}{l}\text { Aspiración } \\
\text { de desarrollo } \\
\text { profesional } \\
\text { (Varianza } \\
\text { explicada: } \\
6.16 \% \text { ) }\end{array}$} & 4.31 & .87 & $\begin{array}{l}13 \text { ¿Cuánto deseas continuar adquiriendo conocimiento para tu } \\
\text { currículum? }\end{array}$ & & & .811 & .659 \\
\hline & 4.59 & .61 & $\begin{array}{l}14 \text { ¿Cuánto deseas continuar aprendiendo en mejorar tus destrezas } \\
\text { docentes? }\end{array}$ & & & .746 & .628 \\
\hline & 4.12 & .88 & 15 ¿Cuánto deseas aprender sobre el actual desarrollo educativo? & & & .722 & .524 \\
\hline & 3.70 & .99 & 16 ¿Cuánto deseas emprender desarrollos profesionales más profundos? & & & .716 & .563 \\
\hline & 4.15 & .86 & 17 ¿Cuánto deseas participar en cursos para tu desarrollo profesional? & & & 690 & .520 \\
\hline
\end{tabular}

Tabla 2. Índices de bondad de ajuste de los modelos propuestos

\begin{tabular}{|c|c|c|c|c|c|c|c|c|}
\hline \multicolumn{2}{|c|}{ Modelos } & $p^{(22)}$ & CMIN/DF & $D F$ & $C F I$ & $T L I$ & RMSEA & SRMR \\
\hline M1 & $\begin{array}{l}\text { Factores } \\
\text { relacionados }\end{array}$ & $<.001$ & 2.009 & 113 & .933 & .934 & .077 & .071 \\
\hline M2 & $\begin{array}{l}\text { Factores } \\
\text { independientes }\end{array}$ & $<.001$ & 3.120 & 119 & .849 & .828 & .111 & .228 \\
\hline
\end{tabular}

Nota. CMIN=razón de chi-cuadrado sobre los grados de libertad; $C F I=$ índice de ajuste comparativo; $T L I=$ =índice de Tucker-Lewis; $R M S E A=$ error cuadrático medio de aproximación; SRMR=raíz cuadrada media residual estandarizada.

varianza. El factor 4, aspiración de desarrollo profesional (5 ítems), explica el $6.16 \%$ de la varianza. Juntos estos cuatro factores evalúan el compromiso profesional y las aspiraciones de desarrollo profesional.

El alfa de Cronbach del total de la escala $(\alpha=.783)$, y de los factores $1(\alpha=.912), 2(\alpha=.733)$, $3(\alpha=.883)$ y $4(\alpha=.787)$ revelaron una adecuada consistencia interna.

\section{Análisis factorial confirmatorio}

La validez del constructo se evaluó mediante un Análisis Factorial Confirmatorio (AFC). El AFC se realizó con la segunda submuestra $\left(n_{2}=178\right)$ sobre los 17 ítems resultantes del AFE. Se pretendía confirmar la estructura de dos factores encontrados en el AFE y si éstos estaban relacionados o eran independientes.

Teniendo en cuenta algunos de los índices de ajuste más utilizados $\left(\chi^{2}, \chi^{2 / g l}, C F I, T L I, R M S E A\right.$ y $S R M R$ ), mediante el método de máxima verosimilitud, se ponen a prueba dos modelos (M): M1: dos factores relacionados; M2: dos factores independientes (Tabla 2). El $\chi^{2}$ debe 
Tabla 3. Correlación factores PECDA

\begin{tabular}{lccc}
\hline & Factor 1 & Factor 2 & Factor 3 \\
\hline Factor 1. Esfuerzo previsto & - & & \\
Factor 2. Persistencia planificada & $.439^{* *}$ & - & \\
Factor 3. Aspiraciones de desarrollo profesional & $.566^{* *}$ & $.216^{* *}$ & - \\
Factor 4. Aspiraciones de liderazgo & .054 & .017 & $.256^{* *}$ \\
\hline$p<.001$ & & &
\end{tabular}

Tabla 4. Método Bootsrap, 1000 muestras con un IC al 95\%

\begin{tabular}{lcccccc}
\hline & Ítems & $\begin{array}{c}\text { Cargas } \\
\text { Factoriales }\end{array}$ & $\begin{array}{c}\text { Media } \\
1000 \text { muestras }\end{array}$ & $\begin{array}{c}\text { Límite } \\
\text { Inferior }\end{array}$ & $\begin{array}{c}\text { Límite } \\
\text { Superior }\end{array}$ & $p$ \\
\hline \multirow{3}{*}{ Esfuerzo previsto } & Ítem 1 & .926 & .923 & .838 & .971 & .003 \\
& Ítem 2 & .866 & .852 & .720 & .948 & .001 \\
& Ítem 3 & .815 & .798 & .649 & .917 & .001 \\
& Ítem 4 & .802 & .783 & .615 & .911 & .001 \\
\hline \multirow{3}{*}{ Aspiraciones de } & Ítem 5 & .862 & .865 & .755 & .935 & .005 \\
liderazgo & Ítem 6 & .845 & .852 & .746 & .915 & .007 \\
& Ítem 7 & .620 & .699 & .386 & .987 & .002 \\
& Ítem 8 & .429 & .600 & .370 & .970 & .001 \\
\hline \multirow{3}{*}{ Persistencia } & Ítem 9 & .942 & .942 & .847 & .985 & .005 \\
planificada & Ítem 10 & .921 & .921 & .868 & .957 & .003 \\
& Ítem 11 & .802 & .801 & .668 & .884 & .003 \\
& Ítem 12 & .670 & .666 & .487 & .816 & .002 \\
\hline \multirow{4}{*}{ Aspiración de } & Ítem 13 & .718 & .721 & .600 & .807 & .004 \\
desarrollo profesional & Ítem 14 & .648 & .641 & .443 & .808 & .002 \\
& Ítem 15 & .682 & .678 & .549 & .780 & .002 \\
& Ítem 16 & .616 & .612 & .492 & .718 & .002 \\
& Ítem 17 & .665 & .658 & .519 & .771 & .002 \\
\hline
\end{tabular}

adquirir valores no significativos ( $p>.05)$ (Jöreskog \& Sörbom, 1989), el $\chi 2 / g l$, se considera aceptable cuando es inferior a 5 (Bentler, 1989), valores superiores a .90 de los índices incrementales ( $C F I$ y $T L I$ ) y mayores o iguales a .08 del RMSEA (Browne \& Cudeck, 1993) y el SRMR (Hu \& Bentler, 1999) se consideran aceptables (Hu \& Bentler, 1995).

En el M2, los índices no se ajustan a valores idóneos, siendo el M1 el que mejores ajustes presenta, con valores de ajuste adecuados. De esta manera, y tal y como se mostrará en el apartado siguiente, se puede concluir que el modelo propuesto por Watt y Richardson (2008; 2013), es generalizable a población española.

Existen relaciones $(p \leq .001)$ entre el factor $1 \mathrm{y}$ los factores 2 y 3 , y entre el factor 3 y los factores 2 y 4 (Tabla 3 ).

El rango de los coeficientes estandarizados de los factores 1 (.802-.926), 2 (.429-.862), 3 (.670$.942)$ y 4 (.695-.712), muestra que los indicadores son consistentes para la medida de ambos constructos
(Tabla 4).

Al mismo tiempo, se aplicó el método bootstrap para calcular la normalidad de los datos, utilizando un total de 1000 muestras con un Intervalo de Confianza (IC) al 95\% (Tabla 4). Este método permite crear un gran número de muestras con reposición de los mismos datos, comprobando así, que los valores obtenidos de las cargas factoriales no están condicionados por la selección de la muestra.

En la Tabla 4, se observa que los valores de las cargas factoriales son muy similares a los valores encontrados en el análisis confirmatorio y se encuentran entre los límites inferiores y superiores del IC al 95\%, siendo, por tanto, todos ellos significativos $(p \leq .007)$.

Asimismo, la consistencia interna de los constructos latentes se calculó mediante la Varianza Media Extractada (VME), la Fiabilidad Compuesta (FC) y la Omega de McDonald $(\Omega)$. Es deseable que los valores de la VME sean 
iguales o superiores a .50 e iguales o superiores a .70 para la FC y $\Omega$.

Los coeficientes de VME, $F C$ y $\Omega$ (>.79), muestran que el modelo de 4 factores relacionados presenta suficientes evidencias de fiabilidad (Tabla 5).

Tabla 5. Valores $V M E, F C$ y $\Omega$ de los factores del PECDA

\begin{tabular}{lccc}
\hline & $V M E$ & $F C$ & $\Omega$ \\
\hline Esfuerzo previsto & .713 & .925 & .944 \\
Persistencia planificada & .629 & .864 & .797 \\
Aspiraciones de desarrollo & .775 & .931 & .920 \\
profesional & & & \\
Aspiraciones de liderazgo & .534 & .851 & 837 \\
\hline
\end{tabular}

\section{Discusión}

El objetivo de este trabajo era validar la escala de compromiso profesional y aspiraciones de desarrollo profesional en una muestra de maestros de enseñanza infantil y primaria y profesores de enseñanza secundaria en España. La hipótesis que se plantea es que la validación de la escala de compromiso profesional y aspiraciones de desarrollo profesional presentará una adecuada validez y fiabilidad en una muestra de maestros y profesores españoles.

En primer lugar, y de acuerdo a esta hipótesis, los resultados de los análisis psicométricos y factoriales confirmatorios realizados, basado en modelos de ecuaciones estructurales mostraron una adecuada estructura factorial, consistencia interna y validez criterial del instrumento. Por consiguiente, se puede indicar que a partir de ahora este cuestionario se puede utilizar para valorar el compromiso profesional y aspiraciones de desarrollo profesional entre los profesionales de la docencia no universitaria. Las propiedades psicométricas halladas son similares a otros instrumentos diseñados $y / o$ validados en el contexto educativo (Álvarez-García et al., 2011; Atxurra et al., 2015; González-Lomelí et al., 2018).

En este sentido, se pudo comprobar que el modelo factorial es recursivo, no hay relaciones de causalidad entre las variables endógenas y los errores de medida no están correlacionados. Además, el análisis factorial mostró cómo los cinco factores obtuvieron saturaciones por encima de .60, indicando una adecuada validez factorial (Varela et al., 2006). Resultados similares fueron encontrados en otros estudios previamente (Watt \& Richardson, 2007; 2008) que analizaban el desarrollo profesional de profesores. Asimismo, mantiene resultados análogos a los encontrados previamente en el análisis de otros constructos en profesores, como son la orientación docente en el profesor universitario (Feixas, 2006).

En cuanto a la fiabilidad del instrumento, se puede observar cómo todos los factores tenían unos índices Alpha de Cronbach por encima de .70 (Nunnally, 1978), lo que indica que los diferentes ítems que agrupa cada factor miden de forma similar, además de mostrar un adecuado ajuste lingüístico a la equivalencia psicológica de los constructos que se deseaban valorar, proporcionando un instrumento de medida psicológico adecuado para el contexto educativo español, similar a otros estudios desarrollados previamente (Castillo et al., 2018). Además, estos resultados son similares a los hallados por Watt y Richardson (2008), quiénes encontraron propiedades psicométricas adecuadas en el instrumento de evaluación de desarrollo profesional en una muestra de 510 profesores de enseñanza primaria y secundaria. Eren y Tezel (2010) también encontraron resultados similares en las propiedades psicométricas de la escala de compromiso profesional y aspiraciones de desarrollo profesional en una muestra de profesores en Turquía. Igualmente, Watt y Richardson (2007) hallaron valores similares en una muestra de profesores australianos en las propiedades psicométricas de la escala. Otros autores como Atxurra y cols. (2015) han hallado resultados consistentes con los nuestros en otras escalas aplicables a la actividad docente, como son los constructos del aprendizaje cooperativo, mostrando la escala de aplicación del aprendizaje cooperativo (CLAS) una adecuada validad y fiabilidad.

De esta manera, se confirma la adecuación del instrumento para valorar el compromiso profesional y aspiraciones de desarrollo profesional en profesores de enseñanza infantil, primaria y secundaria, testando un instrumento en español que puede ayudar a generar un modelo que permita conocer los resultados adaptativos como persistencia, satisfacción, mayor 
compromiso e implicación, etc., dentro de la función docente (Coldwell, 2017), y que se ha demostrado que son relevantes para la mejora de los procesos de enseñanza y aprendizaje del alumnado. Además, se permite testar la importancia del desarrollo profesional entre los docentes, y sus expectativas dentro de su carrera profesional con otros aspectos (i.e. motivación, satisfacción, persistencia y/o calidad en la enseñanza...) que permitan dotar de un carácter empírico a estos constructos tan demandados, pero poco tratados desde un punto de vista científico (Desimone, 2009).

Respecto a las limitaciones del trabajo, se destaca que solamente se ha realizado una valoración transversal de los maestros y profesores, al final de un curso académico, por lo que podrían existir pequeñas diferencias si se realizaran otras medidas de manera longitudinal. Además, se indica que los presentes datos están basados únicamente en medidas percibidas por cada maestro y profesor, por lo que puede ser que no refleje con exactitud sus reales intenciones, pero pueden señalar sus expectativas sobre su profesión en la docencia (Eren, 2012). En este sentido, como prospectiva de futuro se establece el desarrollar varias medidas a diferentes profesores de enseñanza infantil, primaria y secundaria, aumentando la muestra a centros educativos privados, así como a profesores de enseñanza universitaria, con la finalidad de conocer las posibles diferencias que pudieran existir. Además, otros aspectos tan importantes como el carácter interino, vacante, funcionario en prácticas, etc., no se ha tenido en cuenta en este estudio, por lo que, siguiendo las indicaciones de Richter et al. (2011), en cuanto a la relación entre el momento actual de la carrera profesional y el desarrollo profesional, influye en gran medida en la eficacia de los diferentes tipos de desarrollo profesional. Así, estamos seguros que una medición de estas variables en un futuro nos permitiría mejorar la rigurosidad del instrumento.

Como conclusiones de este estudio, se demuestran las propiedades psicométricas de la escala de compromiso profesional y aspiraciones de desarrollo profesional en profesores de enseñanza primaria y secundaria, mostrando una adecuada validez y fiabilidad. De esta manera, gracias a los resultados de esta investigación, podemos disponer de un instrumento útil, fiable y eficaz en contextos educativos, con la finalidad de conocer aspectos tan relevantes en los docentes como son su persistencia, esfuerzo, capacidad de planificación, aspiraciones de liderazgo, etc., dentro de una labor tan importante como es la enseñanza.

\section{Referencias}

Álvarez-García, D., Núñez, J. C., Rodríguez, C., Álvarez, L., \& Dobarro, A. (2011). Propiedades psicométricas del Cuestionario de Violencia Escolar - Revisado (CUVE-R). Revista de Psicodidáctica, 16(1), 59-83.

Anthony, G., \& Ord, K. (2008). Change-of-career secondary teachers: Motivations, expectations and intentions. Asia-Pacific Journal of Teacher Education, 36(4), 359-376.

Atxurra, C., Villardón-Gallego, L., \& Calvete, E. (2015). Diseño y validación de la escala de aplicación del aprendizaje cooperativo (CLAS). Revista de Psicodidáctica, 20(2), 339-357.

Brislin (1970). Back translation for the crosscultural research. Journal of Cross Cultural Research, 1(3), 185-216.

Bruinsma, M., \& Jansen, E. P. W. A. (2010). Is the motivation to become a teacher related to pre-service teachers' intentions to remain in the profession? European Journal of Teacher Education, 33(2), 185-200.

Castillo, I., Solano, S., \& Sepúlveda, A. R. (2018). Validación Mexicana de la Escala de Actitudes Masculinas sobre el Cuerpo en Estudiantes Universitarios Varones. Revista Iberoamericana de Diagnóstico y Evaluación - e Avaliação Psicológica, 46(1), 51-65. doi:10.21865/RIDEP46.1.04

Cavanagh, M. (2011). Students' experiences of active engagement through cooperative learning activities in lectures. Active Learning in Higher Education, 12(1), 23-33. doi:10.1177/1469787410387724

Coldwell, M. (2016). Career orientations and career cultures: Individual and organisational approaches to beginning teachers' careers. Teachers and Teaching, 22(5), 610-624. 
Coldwell, M. (2017). Exploring the influence of professional development on teacher careers: A path model approach. Teaching and Teacher Education, 61, 189-198.

Day, C., \& Gu, Q. (2007). Variations in the conditions for teachers' professional learning and development: Sustaining commitment and effectiveness over a career. Oxford Review of Education, 33(4), 423-443.

Daouk L., \& McDowall A. (2013). Using cognitive interviewing for the semantic enhancement of multi-lingual versions of personality questionnaires. Journal of Personality Assessment, 95(4), 407-416. doi:10.1080/00223891.2012.735300

DeAngelis, K. J., \& Presley, J. B. (2011). Towards a more nuanced understanding of new teacher attrition. Education and Urban Society, 43, 598-626.

Desimone, L. (2009). Improving impact studies of teachers' professional development: Toward better conceptualizations and measures. Educational Researcher, 38(3), 181-199.

Eren, A. (2012). Prospective teachers' future time perspective and professional plans about teaching: The mediating role of academic optimism. Teaching and Teacher Education, 28, 111-123.

Eren, A., \& Tezel, K. (2010). Factors influencing teaching choice, professional plans about teaching, and future time perspective: A mediational analysis. Teaching and Teacher Education, 26, 1416-1428.

Feixas, M. (2006). Cuestionario para el análisis de la acción docente del profesor universitario. Revista de Investigación Educativa, 24(1), 97118.

González-Lomelí, D., Maytorena, M. A., Cárdenas-Niño, L., Tapia-Fonllem, \& C. O. (2018). Perspectiva Temporal de Estudiantes Universitarios Mexicanos y Colombianos. Revista Iberoamericana de Diagnóstico y Evaluación - e Avaliação Psicológica, 46(1), 136-145. doi:10.21865/RIDEP46.1.10

Gillies, R., \& Boyle, M. (2010). Teachers' reflections on cooperative learning: Issues of implementation. Teaching and Teacher Education, 26(4), 933-940. doi:10.1016/j.tate.2009.10.034
Hambleton, R. K. (2001). The next generation of the ITC Test Translation and Adaptation Guidelines. European Journal of Psychological Assessment, 17(3), 164-172.

Hambleton, R. K. (2005). Issues, designs and technical guidelines for adapting test into multiple languages and cultures. En R. Hambleton, P. Merenda y S. Spielberger (Eds.), Adapting educational and psychological test for cross-cultural assessment (pp. 3-38). Filadelfia, NJ: Lawrence Erlbaum Associates.

Malderez, A., Hobson, A. J., Tracey, L., \& Kerr, K. (2007). Becoming a student teacher: Core features of the experience. European Journal of Teacher Education, 30(3), 225-248.

Mau, W. C. J., \& Mau, Y. H. (2006). Factors influencing high school students to persist in aspirations of teaching careers. Journal of Career Development, 32(3), 234-249.

Muñiz, J., Elosua, P., \& Hambleton, R. (2013). Directrices para la traducción y adaptación de los test: Segunda edición. Psicothema, 25(2), 151-157. doi:10.7334/psicothema2013.24

Nunnally, J. C. (1978). Psychometric Theory. Nueva York: McGraw-Hill.

Organization for Economic Co-operation and Development. (2009). Improving equity in education. Informal meeting of OECD ministers of education. Oslo: OECD.

Richardson, P. W., \& Watt, H. M. G. (2006). Who chooses teaching and why? Profiling characteristics and motivations across three Australian universities. Asia-Pacific Journal of Teacher Education, 34(1), 27-56.

Richter, D., Kunter, M., Klusmann, U., Lüdtke, O., \& Baumert, J. (2011). Professional development across the teaching career: Teachers' uptake of formal and informal learning opportunities. Teaching and Teacher Education, 27(1), 116-126.

Rinke, C. R. (2008). Understanding teachers' careers: Linking professional life to professional path. Educational Research Review, 3, 1-13.

Roeser, R. W., \& Galloway, M. G. (2002). Studying motivation to learn in early adolescence: A holistic perspective. En T. Urdan, y F. Pajares (Eds.), Academic motivation of adolescents: Adolescence and 
education. Greenwich, CT: Information Age Publishing.

Spittle, M., Jackson, K., \& Casey, M. (2009). Applying self-determination theory to understand the motivation for becoming a physical education teacher. Teaching and Teacher Education, 25(1), 190-197.

Varela, J., Abalo, J., Rial, A., \& Braña, T. (2006). El análisis factorial confirmatorio de segundo nivel. En J. P. Lévy y J. Varela (Eds.), Modelización con Estructuras de Covarianzas en Ciencias Sociales (pp.239-258). A Coruña: Netbiblo.

Wang, H. H. (2004). Why teach science? Graduate science students' perceived motivations for choosing teaching as a career in Taiwan. International Journal of Science Education, 26(1), 113-128.

Watt, H. M. G., \& Richardson, P. W. (2007). Motivational factors influencing teaching as a career choice: Development and validation of the FIT-choice scale. Journal of Experimental Education, 75(3), 167-202.

Watt, H. M. G., \& Richardson, P. W. (2008). Motivations, perceptions, and aspirations concerning teaching as a career for different types of beginning teachers. Learning and Instruction, 18, 408-428.

Watt, H. M. G., \& Richardson, P. W. (2014). Profiles of professional engagement and career development aspirations among USA preservice teachers. International Journal of Educational Research, 65, 23-40. 